\title{
On a conjecture of Braverman and Kazhdan
}

\author{
S. Cheng, B. C. Ngô
}

\begin{abstract}
In this paper a proof of Conjecture 9.12 of Braverman-Kazhdan in their article $\gamma$-functions of representations and lifting on the acyclicity of their $\ell$-adic $\gamma$-sheaves over certain affine spaces is given for $\operatorname{GL}(n)$.
\end{abstract}

\section{Introduction}

Classical Fourier transforms on vector spaces over local fields and adelic rings have found remarkable connections with the standard $L$-functions $L(s, \pi, \operatorname{std})$ of $\mathrm{GL}(n)$ since Tate [T50] for $n=1$ and Godement-Jacquet GJ72] for general $n$.

More generally, for each reductive group $G$ which is quasi-split over a nonarchimedean local field $F_{v}$ and each representation $\rho$ of its dual group ${ }^{L} G$ satisfying some mild technical conditions, there exists a $\rho$-analogue of the Fourier transform which is essentially the operator of convolution by an invariant distribution $\Phi_{\psi, \rho}$ on $G\left(F_{v}\right)$ where $\psi$ is a fixed additive character of $F_{v}$, whose operator-valued Mellin transform $\mathcal{M}\left(\Phi_{\psi, \rho} *(\bullet)\right)$ is the scalar operator of multiplication by the $\gamma$-function $\gamma_{\psi, \rho}\left(\pi_{v}\right)$ investigated by Braverman-Kazhdan in [BK00]. Similar ideas have been developed further by L. Lafforgue, see for example [L13].

Incarnations $\Phi_{\psi, \rho}$ of $\Phi_{\psi, \rho}$ as $\ell$-adic perverse sheaves over finite fields have been constructed and studied by Braverman-Kazhdan in the last section of [BK00] and subsequently in BK02. The purpose of this paper is to establish Conjecture 9.12 in $\mathrm{BK} 00$ for $\mathrm{GL}(n)$. The argument generalizes that of Braverman-Kazhdan in BK02 for GL(2). Following the classical paradigm the generalization from $\mathrm{GL}(2)$ to $\mathrm{GL}(n)$ involves mirabolic groups as an essential ingredient.

Conventions In this paper $k$ is an algebraic closure of a finite field $k_{0}$ with $q$ elements of characteristic $p$. Let $\ell$ be a prime number which is distinct from $p$, let $\overline{\mathbb{Q}}_{\ell}$ be an algebraic closure of the field of $\ell$-adic numbers. 
If $X$ is a $k$-scheme, let $D_{c}^{b}(X)$ denote the derived category of complexes of $\overline{\mathbb{Q}}_{\ell}$-étale sheaves on $X$ with bounded constructible cohomology, let $[d]$ denote the $d$ th translation functor on $D_{c}^{b}(X)$. If $f$ is a $k$-linear morphism of $k$-schemes, the six functors $f^{*}, f_{*}, f_{!}, f^{!}, \otimes_{X}$ and $\mathcal{H o m}_{X}$ are understood in the derived sense. If $j$ is the morphism of inclusion of an open $k$-subscheme, let $j_{\text {!* }}$ denote the intermediate extension functor of Goresky-MacPherson for $\overline{\mathbb{Q}}_{\ell}$-perverse sheaves (see $\mathrm{BBD} 82$ ).

We will denote by $\mathbb{G}_{a}$ the additive group defined over $k$. It has the ArtinSchreier covering, which is a torsor under the finite group $k_{0}$, given by the Lang isogeny $L_{\mathbb{G}_{a}}: \mathbb{G}_{a} \rightarrow \mathbb{G}_{a}$ with $L_{\mathbb{G}_{a}}(t)=t^{q}-t$. We fix a nontrivial character $\psi: k_{0} \rightarrow \overline{\mathbb{Q}}_{\ell}^{\times}$and denote by $\mathcal{L}_{\psi}$ the rank one $\overline{\mathbb{Q}}_{\ell}$-local system attached to $\psi$ obtained by pushing out the Artin-Schreier covering.

Similarly, we denote by $\mathcal{L}_{c}$ the the rank one $\overline{\mathbb{Q}}_{\ell}$-local system on the multiplicative group $\mathbb{G}_{m}$ attached to a multiplicative character $c: k_{0}^{\times} \rightarrow \overline{\mathbb{Q}}_{\ell}^{\times}$ obtained by pushing out the Kummer covering $L_{\mathbb{G}_{m}}: \mathbb{G}_{m} \rightarrow \mathbb{G}_{m}$ with $L_{\mathbb{G}_{m}}(t)=t^{q-1}$

\section{Contents}

Introduction

1 Katz's hypergeometric sheaves 3

2 Braverman-Kazhdan's $\gamma$-sheaves

3 Conjugation action of the mirabolic group 9

4 Action of $U_{Q}$ by left translation 13

5 Proof of Theorem $2.4 \quad 15$

A The case of parabolic subgroups 18

B Positive hypergeometric sheaves 19

References 
Katz's hypergeometric sheaves

\section{Katz's hypergeometric sheaves}

Let $T$ be a torus defined over $k$ and $\lambda: \mathbb{G}_{m} \rightarrow T$ a nontrivial cocharacter. We note that $\lambda$ is then necessarily a finite morphism so that we have

$$
\Psi(\lambda)=\lambda_{!}\left(j^{*} \mathcal{L}_{\psi}[1]\right)=\lambda_{*}\left(j^{*} \mathcal{L}_{\psi}[1]\right) .
$$

where $j: \mathbb{G}_{m} \rightarrow \mathbb{G}_{a}$ is the inclusion morphism from the multiplicative group into the additive group. We will call $\Psi(\lambda)$ the hypergeometric sheaf attached to $\lambda: \mathbb{G}_{m} \rightarrow T$. They are perverse sheaves on $T$.

Following Katz [K90, Chapter 8], we construct general hypergeometric sheaves on $T$ by convolving the $\Psi(\lambda)$. We recall that convolution products on $T$ are constructed as direct images with respect to the multiplication morphism $\mu: T \times T \rightarrow T$ with $\mu\left(t_{1}, t_{2}\right)=t_{1} t_{2}$. There are two convolution products attached to the direct image functors with or without the compact support condition: for every $\mathcal{F}, \mathcal{G} \in D_{c}^{b}(T)$ we define

$$
\begin{aligned}
\mathcal{F} \star \mathcal{G} & =\mu_{!}(\mathcal{F} \otimes \mathcal{G}) \\
\mathcal{F} * \mathcal{G} & =\mu_{*}(\mathcal{F} \otimes \mathcal{G})
\end{aligned}
$$

related by the morphism of functors

$$
\mathcal{F} \star \mathcal{G} \rightarrow \mathcal{F} * \mathcal{G}
$$

that consists in forgetting the compact support condition. For every collection of possibly repeated nontrivial cocharacters $\underline{\lambda}=\left(\lambda_{1}, \ldots, \lambda_{r}\right)$, we consider the convolution products

$$
\begin{aligned}
& \Psi_{\underline{\lambda}}=\Psi\left(\lambda_{1}\right) \star \cdots \star \Psi\left(\lambda_{r}\right) \\
& \Psi_{\underline{\lambda}}^{*}=\Psi\left(\lambda_{1}\right) * \cdots * \Psi\left(\lambda_{r}\right)
\end{aligned}
$$

and the forget support morphism

$$
\Psi_{\underline{\lambda}} \rightarrow \Psi_{\underline{\lambda}}^{*}
$$

If we denote by $\mathrm{p}_{\underline{\lambda}}: \mathbb{G}_{m}^{r} \rightarrow T$ the homomorphism given by

$$
\mathrm{p}_{\underline{\underline{\lambda}}}\left(t_{1}, \ldots, t_{r}\right)=\prod_{i=1}^{r} \lambda_{i}\left(t_{i}\right)
$$

and by $\operatorname{tr}: \mathbb{G}_{m}^{r} \rightarrow \mathbb{G}_{a}$ the addition morphism $\operatorname{tr}\left(t_{1}, \ldots, t_{r}\right)=\sum_{i=1}^{r} t_{i}$, then

$$
\begin{aligned}
& \Psi_{\underline{\lambda}}=\mathrm{p}_{\underline{\lambda}, !} \operatorname{tr}^{*} \mathcal{L}_{\psi}[r] \\
& \Psi_{\underline{\lambda}}^{*}=\mathrm{p}_{\underline{\lambda}, *} \operatorname{tr}^{*} \mathcal{L}_{\psi}[r] .
\end{aligned}
$$


Braverman-Kazhdan's $\gamma$-sheaves

For $T=\mathbb{G}_{m}$ and $\lambda_{i}: \mathbb{G}_{m} \rightarrow \mathbb{G}_{m}$ being the identity for all $i, \Psi_{\underline{\lambda}}$ is the $r$-fold Kloosterman sheaf considered by Deligne in [D77s]. In general, this is what Braverman and Kazhdan have called $\gamma$-sheaves on tori in BK02.

We will restrict ourselves in a setting where the morphism (11) is an isomorphism. Let $\sigma: T \rightarrow \mathbb{G}_{m}$ be a character. A cocharacter $\lambda: \mathbb{G}_{m} \rightarrow T$ is said to be $\sigma$-positive if the composition $\sigma \circ \lambda: \mathbb{G}_{m} \rightarrow \mathbb{G}_{m}$ is of the form $t \mapsto t^{n}$ where $n$ is a positive integer.

Proposition 1.1. Assume that $\lambda_{1}, \ldots, \lambda_{n}$ are $\sigma$-positive. Then the forgetsupport morphism $\Psi_{\underline{\lambda}} \rightarrow \Psi_{\underline{\lambda}}^{*}$ is an isomorphism. Moreover, $\Psi_{\underline{\lambda}}$ is a perverse local system over the image of $\mathrm{p}_{\underline{\lambda}}$, which is a subtorus of $T$.

Proof. See Appendix B.

Let $\Sigma_{\underline{\lambda}}$ denote the subgroup of the symmetric group $\mathfrak{S}_{r}$ consisting of permutations $\tau \in \mathfrak{S}_{r}$ such that for all $i \in\{1, \ldots, r\}$, we have $\lambda_{\tau(i)}=\lambda_{i}$. This subgroup is of the form $\Sigma_{\underline{\lambda}}=\mathfrak{S}_{r_{1}} \times \cdots \times \mathfrak{S}_{r_{m}}$ where $\left(r_{1}, \ldots, r_{m}\right)$ is the partition of $r$ corresponding to positive number of occurrences in $\left\{\lambda_{1}, \ldots, \lambda_{r}\right\}$.

Proposition 1.2. The group $\Sigma_{\underline{\lambda}}$ acts on $\Psi_{\underline{\lambda}}$ via the sign character.

Proof. This is [D77s, Proposition 7.20].

Proposition 1.3. Let $\mathcal{L}$ be a Kummer local system on $T$. Then if $\lambda_{1}, \ldots, \lambda_{n}$ are $\sigma$-positive, we have

$$
\mathrm{H}_{c}^{i}\left(\Psi_{\underline{\lambda}} \star \mathcal{L}\right)=0
$$

for $i \neq 0$ and $\operatorname{dim} \mathrm{H}_{c}^{0}\left(\Psi_{\underline{\lambda}} \star \mathcal{L}\right)=1$. Moreover, there is a canonical isomorphism

$$
\Psi_{\underline{\lambda}} \star \mathcal{L}=\mathrm{H}_{c}^{0}\left(\Psi_{\underline{\lambda}} \star \mathcal{L}\right) \otimes \mathcal{L} .
$$

Proof. This is BK02, Theorem 4.8].

\section{Braverman-Kazhdan's $\gamma$-sheaves}

Let $G$ be a reductive group over $k$. Let $T$ be a maximal torus of $G, B$ a Borel subgroup containing $T$ and $U$ the unipotent radical of $B$. Let $W=$ $\operatorname{Nor}_{G}(T) / T$ denote the Weyl group of $G, \operatorname{Nor}_{G}(T)$ being the normalizer of $T$ in $G$. The group of cocharacters $\Lambda=\operatorname{Hom}\left(\mathbb{G}_{m}, T\right)$ is a free abelian group of finite type equipped with an action of $W$. The complex dual group $\check{G}$ is equipped with a maximal torus $\check{T}$ and a Borel subgroup $\check{B}$ containing $\check{T}$. We have $\Lambda=\operatorname{Hom}\left(\check{T}, \mathbb{C}^{\times}\right)$. 
We will recall the construction, due to Braverman and Kazhdan, of the $\gamma$-sheaf attached to a representation of the dual group $\check{G}$. Let $\rho: \check{G} \rightarrow$ $\operatorname{GL}\left(V_{\rho}\right)$ be an $r$-dimensional representation of $\check{G}$. The restriction of $\rho$ to $\check{T}$ is diagonalizable i.e. there exists a finite set of weights

$$
\left\{\lambda_{1}, \ldots, \lambda_{m}\right\} \subset \Lambda=\operatorname{Hom}\left(\check{T}, \mathbb{C}^{\times}\right)
$$

such that there is a decomposition into direct sum of eigenspaces

$$
V_{\rho}=\bigoplus_{i=1}^{m} V_{\lambda_{i}}
$$

with $\check{T}$ acting on $V_{\lambda_{i}}$ by the character $\lambda_{i}$. The integers $r_{i}=\operatorname{dim}\left(V_{\lambda_{i}}\right)$ define a partition $r=r_{1}+\cdots+r_{m}$. We will denote

$$
\underline{\lambda}=(\underbrace{\lambda_{1}, \ldots, \lambda_{1}}_{r_{1}}, \ldots, \underbrace{\lambda_{m}, \ldots, \lambda_{m}}_{r_{m}}) \in \Lambda^{r}
$$

where $\lambda_{1}, \ldots, \lambda_{m}$ appear in $\underline{\lambda}$ with multiplicity $r_{1}, \ldots, r_{m}$ respectively.

By choosing a basis $A_{i}=\left\{v_{i, j}, 1 \leq j \leq r_{i}\right\}$ of each $V_{\lambda_{i}}$, we obtain a basis

$$
A=A_{1} \sqcup \ldots \sqcup A_{m}
$$

of $V_{\rho}$. The Weyl group of $\mathrm{GL}\left(V_{\rho}\right)$ can be identified with the symmetric group $\operatorname{Perm}(A)=\mathfrak{S}_{r}$ of permutations of the finite set $A$. Let

$$
\Sigma_{\underline{\lambda}}=\mathfrak{S}_{r_{1}} \times \cdots \times \mathfrak{S}_{r_{m}} \subset \mathfrak{S}_{r}
$$

denote the subgroup consisting of $\tau \in \operatorname{Perm}(A)$ such that $\tau\left(A_{i}\right)=A_{i}$.

Let $\Sigma_{\lambda}^{\prime}$ denote the subgroup of $\operatorname{Perm}(A)$ consisting of permutations $\tau$ such that there exists a permutation $\xi \in \mathfrak{S}_{r}$ such that $\tau\left(A_{i}\right)=A_{\xi(i)}$ for all $i \in\{1, \ldots, m\}$. The application $\tau \mapsto \xi$ defines a homomorphism $\Sigma_{\underline{\lambda}}^{\prime} \rightarrow \mathfrak{S}_{m}$ whose kernel is $\Sigma_{\underline{\lambda}}$. Its image consists of permutations $\xi \in \mathfrak{S}_{m}$ preserving the function $i \mapsto r_{i}$.

The Weyl group $W$ operates on $\Lambda$ and its action preserves the subset $\left\{\lambda_{1}, \ldots, \lambda_{m}\right\}$ of $\Lambda$. It induces a homomorphism $W \rightarrow \mathfrak{S}_{m}$. Its image is contained in the subgroup of $\mathfrak{S}_{m}$ of permutations preserving the function $i \mapsto r_{i}$ so that there is a canonical homomorphism

$$
\rho_{W}: W \rightarrow \Sigma_{\underline{\lambda}}^{\prime} / \Sigma_{\underline{\lambda}}
$$


Braverman-Kazhdan's $\gamma$-sheaves

We derive an extension $W^{\prime}$ of $W$ by $\Sigma_{\underline{\lambda}}$ fitting into the diagram

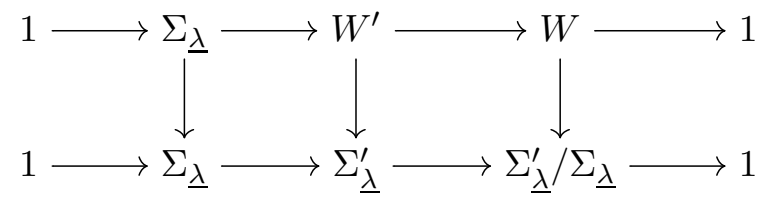

where an element $w^{\prime} \in W^{\prime}$ consists of a pair $(w, \xi)$ with $w \in W$ and $\xi \in \Sigma_{\underline{\lambda}}^{\prime}$ such that $\rho_{W}(w)=\xi \bmod \Sigma_{\underline{\lambda}}$. One can check that the homomorphism $\mathrm{p}_{\underline{\lambda}}: \mathbb{G}_{m}^{r} \rightarrow T$, and its dual $\left.\rho\right|_{T^{\prime}}: \check{T} \rightarrow\left(\mathbb{C}^{\times}\right)^{r}$, are $W^{\prime}$-equivariant.

As in Section 1, the finite sequence of $\sigma$-positive weights $\underline{\lambda} \in \Lambda^{r}$ gives rise to a hypergeometric sheaf $\Psi_{\underline{\lambda}}$ on $T$, equipped with an action of $\Sigma_{\underline{\lambda}}$. This hypergeometric sheaf is well behaved under certain positivity condition that can be phrased in the present circumstance as follows. Let $\sigma: G \rightarrow \mathbb{G}_{m}$ be a character of $G$, we also denote $\sigma: \mathbb{C}^{\times} \rightarrow \check{G}$ the dual cocharacter. A representation $\rho: \check{G} \rightarrow \operatorname{GL}\left(V_{\rho}\right)$ is said to be $\sigma$-positive if for every weight $\lambda_{i}$ occurring $V_{\rho}, \lambda_{i} \circ \sigma: \mathbb{C}^{\times} \rightarrow \mathbb{C}^{\times}$is of the form $t \mapsto t^{n}$ where $n$ is a positive integer. We will assume that $\rho$ is $\sigma$-positive. We will also assume that the homomorphism $\mathrm{p}_{\underline{\lambda}}: \mathbb{G}_{m}^{r} \rightarrow T$ is surjective. Under these assumptions, we know that $\Psi_{\underline{\lambda}}$ is a local system on $T$ with the degree shift $[\operatorname{dim}(T)]$.

As the homomorphism $\mathrm{p}_{\underline{\lambda}}: \mathbb{G}_{m}^{r} \rightarrow T$ is $W^{\prime}$-equivariant and the morphism $\operatorname{tr}: \mathbb{G}_{m}^{r} \rightarrow \mathbb{G}_{a}$ is invariant under the action of $W^{\prime}$, we have an action of $W^{\prime}$ on the hypergeometric sheaf $\Psi_{\underline{\lambda}}=\mathrm{p}_{\underline{\lambda}, !} \operatorname{tr}^{*} \mathcal{L}_{\psi}[r]$ compatible with the action of $W^{\prime}$ on $T$ via $W^{\prime} \rightarrow W$ : for every $w^{\prime}=(w, \xi)$ with $w \in W$ and $\xi \in \Sigma_{\underline{\lambda}}^{\prime}$ having the same image in $\Sigma_{\underline{\lambda}}^{\prime} / \Sigma_{\underline{\lambda}}$, by [BK02, Proposition 6.2] we have an isomorphism,

$$
\iota_{w^{\prime}}^{\prime}: w^{*} \Psi_{\underline{\lambda}} \rightarrow \Psi_{\underline{\lambda}} .
$$

We also know that the restriction of this action to $\Sigma_{\underline{\lambda}}$ is the sign character i.e. for $w^{\prime}=(1, \xi)$ with $\xi \in \Sigma_{\underline{\lambda}}$, we have $\iota_{w^{\prime}}^{\prime}=\operatorname{sign}(\bar{\xi}), \xi$ being considered as a permutation of the finite set $A$. For every $w^{\prime}=(w, \xi)$ we set

$$
\iota_{w^{\prime}}=\operatorname{sign}_{r}(\xi) \operatorname{sign}_{W}(w) \iota_{w^{\prime}}^{\prime}: w^{*} \Psi_{\underline{\lambda}} \rightarrow \Psi_{\underline{\lambda}}
$$

where $\operatorname{sign}_{r}: \mathfrak{S}_{r} \rightarrow\{ \pm 1\}$ and $\operatorname{sign}_{W}: W \rightarrow\{ \pm 1\}$ are the sign characters of $\mathfrak{S}_{r}$ and $W$ respectively. We can then check that $\iota_{w^{\prime}}$ depends only on $w$ so that we get an action of $W$ on $\Psi_{\underline{\lambda}}$.

Now we recall the Grothendieck-Springer simultaneous resolution of the 
fibers of the Steinberg morphism (see [S65, Section 6]) $c: G \rightarrow S=T / W$ :

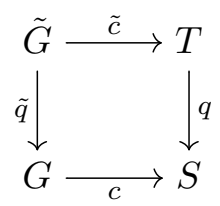

where $\tilde{G}$ is the variety of pairs $(g, h) \in G \times G / B$ such that $h^{-1} g h \in B$, the morphism $\tilde{c}$ given by $(g, h) \mapsto h^{-1} g h \bmod U$ is smooth, and the morphism $\tilde{q}$ given by $(g, h) \mapsto g$ is proper and small in the sense of Goresky-MacPherson. If $T^{\mathrm{rss}}$ is the largest open subset of $T$ where $W$ acts freely and $S^{\mathrm{rss}}=T^{\mathrm{rss}} / W$, then the diagram is Cartesian over $S^{\text {rss }}$. In particular $\tilde{G}^{\text {rss }} \rightarrow G^{\text {rss }}$ is a $W$ torsor, where $j^{\text {rss }}: G^{\text {rss }} \rightarrow G$ denotes the base change of the inclusion morphism $S^{\text {rss }} \subset S$ to $G$.

Recall that the induction functor $\operatorname{Ind}_{T}^{G}: D_{c}^{b}(T) \rightarrow D_{c}^{b}(G)$ is defined by

$$
\operatorname{Ind}_{T}^{G}(\mathcal{F})=\tilde{q}_{!} \tilde{c}^{*} \mathcal{F}[d]
$$

where $d=\operatorname{dim}(G)-\operatorname{dim}(T)$. Because $q$ is a small map and $\Psi_{\underline{\lambda}}$ is a perverse local system on $T$,

$$
\operatorname{Ind}_{T}^{G}\left(\Psi_{\underline{\lambda}}\right)=\tilde{q}_{!} \tilde{c}^{*} \Psi_{\underline{\lambda}}[d]
$$

is a perverse sheaf isomorphic to the intermediate extension of its restriction to $G^{\text {rss }}$ :

$$
\operatorname{Ind}_{T}^{G}\left(\Psi_{\underline{\lambda}}\right)=j_{! *}^{\mathrm{rss}} j^{\mathrm{rss}, *} \operatorname{Ind}_{T}^{G}\left(\Psi_{\underline{\lambda}}\right) .
$$

For $\tilde{G}^{\text {rss }} \rightarrow G^{\text {rss }}$ is a $W$-torsor and $\Psi_{\underline{\lambda}}$ is $W$-equivariant, $W$ operates on $j^{\text {rss,*}} \operatorname{Ind}_{T}^{G}\left(\Psi_{\underline{\lambda}}\right)$. By functoriality of the intermediate extension functor, this action of $W$ can be extended to the perverse sheaf $\operatorname{Ind}_{T}^{G}\left(\Psi_{\underline{\lambda}}\right)$.

Definition 2.1. The $\gamma$-sheaf attached to $\rho$ is the $W$-invariant direct factor of the perverse sheaf $\operatorname{Ind}_{T}^{G}\left(\Psi_{\underline{\lambda}}\right)$

$$
\Phi_{\rho}=\operatorname{Ind}_{T}^{G}\left(\Psi_{\underline{\lambda}}\right)^{W} .
$$

As a direct factor of $\operatorname{Ind}_{T}^{G}\left(\Psi_{\underline{\lambda}}\right), \Phi_{\rho}$ is also isomorphic to the intermediate extension of its restriction to $G^{\text {rss }}$. There is thus a slightly different way to construct it: we start by descending the restriction of $\Psi_{\underline{\lambda}}$ to $T^{\mathrm{rss}}$, which is a $W$-equivariant perverse local system on $T^{\text {rss }}$, to a perverse local system $\Phi_{\underline{\lambda}, S^{\mathrm{rss}}}$ on $S^{\mathrm{rss}}$. Then we have

$$
\Phi_{\rho}=j_{! *}^{\mathrm{rss}} c^{\mathrm{rss}, *} \Phi_{\underline{\lambda}, S^{\mathrm{rss}}}[d]
$$


The induction functor admits a left adjoint $\operatorname{Res}_{T}^{G}: D_{c}^{b}(G) \rightarrow D_{c}^{b}(T)$, the restriction functor, defined by

$$
\operatorname{Res}_{T}^{G}(\mathcal{F})=\pi_{!} i^{*}(\mathcal{F})
$$

where $\pi: B \rightarrow B / U=T$ and $i: B \rightarrow G$ denote the quotient and inclusion morphisms. More generally $\operatorname{Res}_{M}^{G}: D_{c}^{b}(G) \rightarrow D_{c}^{b}(M)$ could be defined if we replace $B$ by a standard parabolic subgroup $P$ of $G$ and $T$ by the Levi component $M$ of $P$ in (3). The adjunction between restriction and induction and Frobenius reciprocity imply the following

Proposition 2.2. Let $\Phi_{\rho, M}$ denote the perverse sheaf $\Phi_{\rho^{\prime}}$ on $M$ where $\rho^{\prime}$ denotes the restriction of $\rho$ from $\check{G}$ to $\check{M}$, then

$$
\Phi_{\rho, M} \simeq \operatorname{Res}_{M}^{G}\left(\Phi_{\rho}\right) .
$$

Proof. This is the first statement of [BK02, Theorem 6.6].

In [BK00, Conjecture 9.2] Braverman and Kazhdan have conjectured the following vanishing property of $\Phi_{\rho}$.

Conjecture 2.3. Let $\pi_{U}: G \rightarrow G / U$ denote the quotient map. For every $\sigma$-positive representation $\rho$ of $\breve{G}, \pi_{U, !} \Phi_{\rho}$ is supported on the closed subset $T=B / U$ of $G / U$.

The conjecture can be reformulated as follows. For every geometric point $g \in G-B$, we conjecture that

$$
\mathrm{H}_{c}^{*}\left(g U, i^{*} \Phi_{\rho}\right)=0
$$

where $i: g U \rightarrow G$ denotes the inclusion map.

Braverman and Kazhdan have verified their conjecture for groups of semisimple rank one, and for $G=\mathrm{GL}(n), \sigma=\operatorname{det}$ and $\rho$ the standard representation of $\mathrm{GL}_{n}(\mathbb{C})$.

Theorem 2.4. The above conjecture holds for $G=\mathrm{GL}(n), \sigma=\operatorname{det}$ and an arbitrary $\sigma$-positive representation of $\rho$ of $\mathrm{GL}_{n}(\mathbb{C})$.

Our argument applies in the case when $\mathrm{p}_{\underline{\lambda}}: \mathbb{G}_{m}^{r} \rightarrow T$ is surjective, however for $\sigma$-positive $\rho$ the only other possibility is when $\rho$ factors through det $: \mathrm{GL}_{n}(\mathbb{C}) \rightarrow \mathbb{C}^{\times}$, which implies that $\Phi_{\rho}$ is supported on the center of $\mathrm{GL}(n)$, so the theorem holds trivially in this case as well. 


\section{Conjugation action of the mirabolic group}

Our proof of the Braverman-Kazhdan conjecture for $G=\mathrm{GL}(n)$ is based on the geometry of the conjugation action of the mirabolic. This geometry has been described by Bernstein in [B84, 4.1-4.2]. What we aim for here is to put Bernstein's description into a form suitable for our purpose. For consistency of notations with B84 we will follow Bernstein and consider left group actions and left quotients for the rest of this paper. In particular our version of Theorem 2.4 applies to $U \backslash G$ instead of $G / U$.

Let $V$ be the standard $n$-dimension $k$-vector space with the standard basis $e_{1}, \ldots, e_{n}$. We consider the filtration $0 \subset F_{1} \subset F_{2} \subset \cdots \subset F_{n}=V$ where $F_{i}$ is the subvector space generated by $e_{1}, \ldots, e_{i}$. We will denote $E_{i}=V / F_{i}$.

We denote by $Q$ the mirabolic subgroup of $G=\mathrm{GL}(V)$ consisting of elements $g \in G$ fixing the line generated by $e_{1}$, and $Q_{1}$ the subgroup consisting of elements $g \in G$ fixing the vector $e_{1}$. We consider the $Q$-equivariant stratification of $G$

$$
G=\bigsqcup_{m=1}^{n} X_{m}
$$

where $X_{m}$ is the locally closed subset of $V$ consisting of $g \in G$ such that the subspace $F_{x}$ of $V$ generated by the vectors $v, x v, x^{2} v, \ldots$ is of dimension $m$. We will prove that $\left[Q_{1} \backslash X_{m}\right]$ "looks like" a similar quotient $\left[Q_{1} \backslash G\right]$ in lower rank. This statement can be made precise as follows.

Theorem 3.1. There exists a smooth surjective morphism

$$
\phi_{m}:\left[\mathrm{GL}(n-m) \backslash\left(\mathbb{A}^{m} \times \mathrm{GL}(n-m) \times \mathbb{A}^{n-m}\right)\right] \rightarrow\left[Q_{1} \backslash X_{m}\right]
$$

where $g \in \mathrm{GL}_{n-m}$ acts on $\left(a, x^{\prime}, v\right) \in \mathbb{A}^{m} \times \mathrm{GL}(n-m) \times \mathbb{A}^{n-m}$ by the formula

$$
g\left(a, x^{\prime}, v\right)=\left(a, g x^{\prime} g^{-1}, v g^{-1}\right)
$$

Moreover, if $\phi_{m}\left(a, x^{\prime}, v\right)=x$ then

$$
c(x)=a_{t} c\left(x^{\prime}\right)
$$

where $c(x)$ and $c\left(x^{\prime}\right)$ are the characteristic polynomials of $x$ and $x^{\prime}$ respectively, written with the formal variable $t$, and where $a_{t}$ is the polynomial

$$
a_{t}=t^{m}+a_{1} t^{m-1}+\cdots+a_{m}
$$

for every $a=\left(a_{1}, \ldots, a_{m}\right) \in \mathbb{A}^{m}$. 
Theorem 3.1 implies, through an induction on the rank $n$, that the mirabolic group acts on the space of matrices $x \in G$ of a given characteristic polynomial with finitely many orbits.

Proof. For each $m$, we have a fibration

$$
\pi_{m}: X_{m} \rightarrow \mathrm{Gr}_{m}
$$

where $\mathrm{Gr}_{m}$ is the Grassmannian of $m$-dimensional subspaces $F_{x}$ of $V$ containing $v$. It maps $x \in X_{m}$ to the subspace $F_{x}$ of $V$ generated by the vectors $v, x v, x^{2} v, \ldots$ which is of dimension $m$.

For each $m$ we also have the subspace $F_{m}$ and the quotient space $E_{m}$ of $V$. When there is no possible confusion on $m$, we will drop the index $m$ and write simply $F$ for $F_{m}, E$ for $E_{m}$. We consider the parabolic subgroup $P$ of $G$ consisting of elements $g \in G$ such that $g F_{m}=F_{m}$. An element $g \in P$ can be described as a block matrix

$$
g=\left[\begin{array}{cc}
g_{F} & v \\
0 & g_{E}
\end{array}\right]
$$

where $g_{F} \in \mathrm{GL}(F)$ and $g_{E} \in \mathrm{GL}(E)$. The group $Q_{1}$ acts transitively on $\mathrm{Gr}_{m}$ and its stabilizer at a point $F \in \mathrm{Gr}_{m}$ is $P \cap Q_{1}$. The intersection $P \cap Q_{1}$ can be described by the condition $g_{F} \in Q_{F, 1}$ where $Q_{F, 1}$ is the subgroup of $\operatorname{GL}(F)$ defined by $g_{F} e_{1}=e_{1}$.

The fiber $\pi_{m}^{-1}(F)$ is the open subset of $P$ consisting of block matrices

$$
x=\left[\begin{array}{cc}
x_{F} & y \\
0 & x_{E}
\end{array}\right]
$$

such that $v$ is a cyclic vector of $F$ with respect to the action of $x_{F}$. The group $P \cap Q_{1}$ acts on $\pi_{m}^{-1}(F)$ by conjugation and we have

$$
X_{m}=\pi_{m}^{-1}(F) \times{ }^{P \cap Q_{1}} Q_{1} .
$$

For $x_{F}$ as above, $v, x_{F} v, \ldots, x_{F}^{m-1} v$ form a basis of $F$. It follows that there exists a unique $g_{F} \in Q_{F, 1}$ such that $g_{F}^{-1} x_{F} g_{F}$ has the form of a companion matrix

$$
\left[\begin{array}{ccc|c}
0 & \cdots & 0 & -a_{m} \\
\hline & & -a_{m-1} \\
& \mathrm{I}_{m-1} & & \vdots \\
& & & -a_{1}
\end{array}\right]
$$


where $\mathrm{I}_{m-1}$ is the the identity matrix of size $m-1$, and $a_{1}, \ldots, a_{m}$ are the coefficients of the characteristic polynomial of $x_{F}$. It follows that

$$
X_{m}=Y_{m} \times{ }^{H_{m}} Q_{1}
$$

where $Y_{m}$ is the space of matrices of the form

$$
x=\left[\begin{array}{cc}
x_{F} & y \\
0 & x_{E}
\end{array}\right]
$$

where $x_{F}$ is a companion matrix as in (5), $H_{m}$ is the subgroup of $P$ of matrices of the form

$$
h=\left[\begin{array}{cc}
\mathrm{I}_{F} & v \\
0 & g_{E}
\end{array}\right]
$$

acting on $Y_{m}$ by conjugation. The group $H_{m}$ has the structure of a semidirect product

$$
H_{m}=U_{P} \rtimes \mathrm{GL}(E)
$$

where $U_{P}$, the unipotent radical of $P$, consists of matrices of the form

$$
u=\left[\begin{array}{cc}
\mathrm{I}_{F} & v \\
0 & \mathrm{I}_{E}
\end{array}\right]
$$

It will be convenient to regard $u-\mathrm{I}_{V}$ as a linear application $v \in \operatorname{Hom}(E, F)$.

The action of $U_{P}$ on $X_{F}$ can be written down as follows

$$
\left[\begin{array}{cc}
\mathrm{I}_{F} & -v \\
0 & \mathrm{I}_{E}
\end{array}\right]\left[\begin{array}{cc}
x_{F} & y \\
0 & x_{E}
\end{array}\right]\left[\begin{array}{cc}
\mathrm{I}_{F} & v \\
0 & \mathrm{I}_{E}
\end{array}\right]=\left[\begin{array}{cc}
x_{F} & y+x_{F} v-v x_{E} \\
0 & x_{E}
\end{array}\right] .
$$

In other words, the action of $v \in \operatorname{Hom}(E, F)$ on the variable $y$ consists in a translation by $x_{F} v-v x_{E}$.

Now, the rank of the linear transformation of $\operatorname{Hom}(E, F)$ given by

$$
v \mapsto x_{F} v-v x_{E}
$$

depends on the number of common eigenvalues of $x_{F}$ and $x_{E}$, in particular it is an isomorphism if $x_{F}$ and $x_{E}$ have no common eigenvalues. Thus if $x_{F}$ and $x_{E}$ have no common eigenvalues, $U_{P}$ acts simply transitively on the fiber of $Y_{m}$ over $\left(x_{F}, x_{E}\right)$ by conjugation. However, for our purpose, we will need a statement uniform with respect to $\left(x_{F}, x_{E}\right)$, no matter whether they have common eigenvalues or not. 
Lemma 3.2. Let $0=F_{0} \subset F_{1} \subset F_{2} \subset \cdots \subset F_{m-1} \subset F$ be a filtration of $F$ with $\operatorname{dim}\left(F_{j}\right)=j$. Let $x_{F} \in \mathrm{GL}(F)$ be a linear transformation such that $x_{F}\left(F_{j}\right) \subset F_{j+1}$ and the induced application $F_{j} / F_{j-1} \rightarrow F_{j+1} / F_{j}$ is an isomorphism for every $j$ in the range $1 \leq j \leq m-1$. Let $x_{E} \in G L(E)$ be an arbitrary linear transformation.

We consider two subgroups of $U_{P}=\operatorname{Hom}(E, F)$ :

- $U_{1}=\operatorname{Hom}\left(E, F_{1}\right)$.

- $U_{m-1}=\operatorname{Hom}\left(E, F_{m-1}\right)$.

Then the action of $U_{1} \times U_{m-1}$ on the space of matrices of the form

$$
x=\left[\begin{array}{cc}
x_{F} & y \\
0 & x_{E}
\end{array}\right]
$$

given by

$$
\left(u_{1}, u_{m-1}\right) x=u_{1} u_{m-1} x u_{m-1}^{-1}
$$

is simply transitive.

Proof. We write

$$
u_{1}=\left[\begin{array}{cc}
\mathrm{I}_{F} & v_{1} \\
0 & \mathrm{I}_{E}
\end{array}\right] \text { and } u_{m-1}=\left[\begin{array}{cc}
\mathrm{I}_{F} & v_{m-1} \\
0 & \mathrm{I}_{E}
\end{array}\right]
$$

with $u_{1} \in \operatorname{Hom}\left(E, F_{1}\right)$ and $u_{m-1} \in \operatorname{Hom}\left(E, F_{m-1}\right)$. Then we have

$$
u_{1} u_{m-1} x u_{m-1}^{-1}=\left[\begin{array}{cc}
x_{F} & y+v_{1}+v_{m-1} x_{E}-x_{F} v_{m-1} \\
0 & x_{E}
\end{array}\right]
$$

The lemma is now equivalent to saying that the linear application

$$
\operatorname{Hom}\left(E, F_{1}\right) \times \operatorname{Hom}\left(E, F_{m-1}\right) \rightarrow \operatorname{Hom}(E, F)
$$

given by $\left(v_{1}, v_{m-1}\right) \mapsto v_{1}+v_{m-1} x_{E}-x_{F} v_{m-1}$ is an isomorphism. This is equivalent to proving that the map

$$
A=\operatorname{Hom}\left(E, F_{m-1}\right) \rightarrow \operatorname{Hom}\left(E, F / E_{1}\right)=B
$$

given by

$$
\phi\left(v_{m-1}\right)=v_{m-1} x_{E}-x_{F} v_{m-1} \bmod \operatorname{Hom}\left(E, F_{1}\right)
$$

is an isomorphism. 
Action of $U_{Q}$ by left translation

We consider the filtration $0 \subset A_{1} \subset \cdots \subset A_{m-1}=A$ with $A_{j}=$ $\operatorname{Hom}\left(E, F_{j}\right)$ and the filtration $0 \subset B_{1} \subset \cdots \subset B_{m-1}=B$ with $B_{j}=$ $\operatorname{Hom}\left(E, F_{j+1} / F_{1}\right)$. We observe that $\phi\left(A_{j}\right) \subset B_{j}$ for all $j$ and the induced map on the associated graded $A_{j} / A_{j-1} \rightarrow B_{j} / B_{j-1}$ is an isomorphism. Indeed the linear application $\phi_{E}: v_{m-1} \mapsto v_{m-1} x_{E}$ satisfies $\phi_{E}\left(A_{j}\right) \subset B_{j-1}$ and hence induces the zero map on the associated graded $A_{j} / A_{j-1} \rightarrow$ $B_{j} / B_{j-1}$. On the other hand, $\phi_{F}: v_{m-1} \mapsto x_{F} v_{m-1}$ satisfies $\phi_{F}\left(A_{j}\right) \subset B_{j}$ and induces an isomorphism on the associated graded $A_{j} / A_{j-1} \rightarrow B_{j} / B_{j-1}$ by assumption on $x_{F}$. It follows that $\phi$ is an isomorphism.

We infer from the lemma the existence of a canonical isomorphism

$$
Y_{m}=\mathbb{A}^{m} \times \mathrm{GL}(E) \times \operatorname{Hom}\left(E, F_{1}\right) \times \operatorname{Hom}\left(E, F_{m-1}\right)
$$

mapping $x \mapsto\left(x_{F}, x_{E}, v_{1}, v_{m-1}\right)$ with $x_{F}$ a companion matrix as in (5)), $x_{E} \in \mathrm{GL}(E), v_{1} \in \operatorname{Hom}\left(E, F_{1}\right)$ and $v_{m-1} \in \operatorname{Hom}\left(E, F_{m-1}\right)$ such that

$$
x=\left[\begin{array}{cc}
\mathrm{I}_{F} & v_{1} \\
0 & \mathrm{I}_{E}
\end{array}\right]\left[\begin{array}{cc}
\mathrm{I}_{F} & v_{m-1} \\
0 & \mathrm{I}_{E}
\end{array}\right]\left[\begin{array}{cc}
x_{F} & 0 \\
0 & x_{E}
\end{array}\right]\left[\begin{array}{cc}
\mathrm{I}_{F} & -v_{m-1} \\
0 & \mathrm{I}_{E}
\end{array}\right] .
$$

In these new coordinates, the action of the subgroup $U_{m-1} \rtimes \operatorname{GL}(E)$ of $H_{m}=U_{P} \rtimes \mathrm{GL}(E)$ can be described as follows: the action $\left(v_{m-1}^{\prime}, g_{E}\right) \in$ $\operatorname{Hom}\left(E, F_{m-1}\right) \rtimes \mathrm{GL}(E)$ on $x=\left(x_{F}, x_{E}, v_{1}, v_{m-1}\right)$ is given by:

$$
\left(v_{m-1}^{\prime}, g_{E}\right) x=\left(x_{F}, g_{E} x_{E} g_{E}^{-1}, v_{1} g_{E}^{-1}, v_{m-1}+v_{m-1}^{\prime}\right) .
$$

One should note that $U_{m-1} \rtimes \mathrm{GL}(E)$ is not a normal subgroup of $U_{P} \rtimes \mathrm{GL}(E)$ and the action of the full $U_{P}$ is unfortunately very complicated in these coordinates. Nevertheless, we have a smooth surjective morphism

$$
\left[\left(U_{m-1} \rtimes \mathrm{GL}(E) \backslash Y_{m}\right] \rightarrow\left[H_{m} \backslash Y_{m}\right] .\right.
$$

This completes the proof of Theorem 3.1.

\section{Action of $U_{Q}$ by left translation}

The unipotent radical $U_{Q}$ of the mirabolic group $Q$ consists of matrices of the form

$$
u=\left[\begin{array}{c|c}
1 & v \\
\hline 0 & \mathrm{I}_{n-1}
\end{array}\right]
$$

where $v \in \operatorname{Hom}\left(E_{1}, F_{1}\right)$. The action of $U_{Q}$ on $G$ by left translation $g \mapsto$ $u g$ respects the stratification $G=\bigsqcup_{m} X_{m}$. In this section, we will pay 
Action of $U_{Q}$ by left translation

particular attention to the evaluation of the characteristic polynomial on left cosets of $U_{Q}$ i.e. the function $u \mapsto c(u x)$ for $x$ in each stratum $X_{m}$.

The characteristic polynomial $c(x)$ of $x \in G$, with formal variable $t$, is of the form $c(u x)=t^{n}+a_{1} t^{n-1}+\cdots+a_{n}$ where $a_{1}, \ldots, a_{n}$ are $G$-invariant functions of $x$. It can be regarded as a morphism $c: G \rightarrow \mathbb{A}^{n}$ with $c(u x)=$ $\left(a_{1}, \ldots, a_{n}\right)$.

Proposition 4.1. For every $x \in G$, the morphism $l_{x}: U_{Q}=\operatorname{Hom}\left(E_{1}, F_{1}\right) \rightarrow$ $\mathbb{A}^{n}$ given by $l_{x}(u)=c(u x)-c(x)$ is linear. If $x \in X_{m}$, the linear application $l_{x}$ is of rank $m-1$.

For every $x \in X_{m}$ is $Q$-conjugate to a matrix of the form (6) with $x_{F}$ being a companion matrix as in (5), we can assume that the matrix $x$ is of this special form. In particular we have $x \in P$ where $P$ the parabolic group which preserves the subspace $F_{m}$. Let $U_{P}$ and $L_{P}$ denote respectively its unipotent radical and the standard Levi component. Every element $u \in U_{Q}$ can be written uniquely in the form $u=u_{L} u_{U}$ where $u_{L} \in U_{Q} \cap L_{P}$ and $u_{U} \in U_{Q} \cap U_{P}$ where $u_{L}$ is a matrix of the form

$$
u_{L}=\left[\begin{array}{c|c|c}
1 & v & 0 \\
\hline 0 & I_{m-1} & 0 \\
\hline 0 & 0 & I_{n-m}
\end{array}\right]
$$

where $v=\left(v_{1}, \ldots, v_{m-1}\right) \in \mathbb{A}^{m-1}$ is a row vector. The proposition can now be derived from a matrix calculation.

Lemma 4.2. For $x \in X_{m}$ of the form (6) with $x_{F}$ being a companion matrix as in (5) and $u \in U_{Q}$ with $u=u_{L} u_{U}$ as above, we have

$$
c(u x)=c\left(u_{L} x_{F}\right) c\left(x_{E}\right) .
$$

Moreover, if we write $u_{L}$ in coordinates $\left(v_{1}, \ldots, v_{m-1}\right) \in V=\mathbb{A}^{m-1}$ as above, and write $c\left(u_{L} x_{F}\right)-c_{L}\left(x_{F}\right)$ in coordinates $\left(a_{1}, \ldots, a_{m}\right) \in A=\mathbb{A}^{m}$ that are coefficients of the characteristic polynomials, then the application $u_{L} \mapsto c\left(u_{L} x_{F}\right)-c_{L}\left(x_{F}\right)$ induces an linear isomorphism between $V$ and the subspace of $A$ defined by the equation $a_{m}=0$.

Proof. By direct calculation, we find the following formula for the characteristic polynomial of

$$
u_{L} x_{F}=\left[\begin{array}{c|ccc}
1 & -v_{1} & \cdots & -v_{m-1} \\
\hline 0 & & & \\
\vdots & & I_{m-1} & \\
0 & & &
\end{array}\right]\left[\begin{array}{ccc|c}
0 & \cdots & 0 & -a_{m} \\
\hline & & -a_{m-1} \\
& \mathrm{I}_{m-1} & \vdots \\
& & & -a_{1}
\end{array}\right] .
$$


Proof of Theorem 2.4

We have

$$
c\left(u_{L} x_{F}\right)=t^{m}+b_{1} t^{m-1}+\cdots+b_{m-1} t+b_{m}
$$

where $a_{m}=b_{m}$ and for $1 \leq r \leq m-1$

$$
b_{r}=a_{r}+\sum_{i=1}^{r-1} a_{i} v_{r-i}+v_{r} .
$$

The lemma follows.

\section{Proof of Theorem 2.4}

We will deduce Theorem 2.4 from the analogous statement for the mirabolic subgroup $Q$ that ( $\dagger)$ if $g \in G-Q$, then $\mathrm{H}_{c}^{*}\left(U_{Q} g,\left.\Phi_{\rho}\right|_{U_{Q} g}\right)=0$.

To this end take $g \in G-B$, there are two cases: $g \in Q$ or $g \notin Q$.

If $g \notin Q$, by $(\dagger)$ we know that $\mathrm{H}_{c}^{*}\left(U_{Q} g,\left.\Phi_{\rho}\right|_{U_{Q} g}\right)=0$. In fact for all $u \in$ $U_{B}, u g \notin Q$ so that more generally we have $\mathrm{H}_{c}^{*}\left(U_{Q} u g,\left.\Phi_{\rho}\right|_{U_{Q} u g}\right)=0$ for all $u \in U_{B}$. Now one can establish the vanishing of $\mathrm{H}_{c}^{*}\left(U_{B} g, i^{*} \Phi_{\rho}\right)$ by using the Leray spectral sequence associated with the morphism $U_{B} g \rightarrow U_{Q} \backslash\left(U_{B} g\right)$.

Now we consider the case $g \in Q$. Let $L_{Q}$ denote the standard Levi factor of $Q, g_{L}$ the image of $g$ in $L_{Q}$. We have $g_{L} \notin B \cap L_{Q}$. Using the definition of the restriction functor, we have

$$
\mathrm{H}_{c}^{*}\left(U_{B} g, i^{*} \Phi_{\rho}\right)=\mathrm{H}_{c}^{*}\left(\left(U_{B} \cap L\right) g_{L},\left.\operatorname{Res}_{L}^{G}\left(\Phi_{\rho}\right)\right|_{\left(U_{B} \cap L\right) g_{L}}\right)
$$

where $\operatorname{Res}_{L}^{G}\left(\Phi_{\rho}\right)=\Phi_{L, \rho}$ by Proposition 2.2. At this point we can conclude by an induction argument.

It remains to establish $(\dagger)$. For convenience of induction we will prove the following equivalent proposition:

Proposition 5.1. Let $G$ be a direct product of general linear groups and $Q$ a mirabolic subgroup of $G$ of the form

$$
Q=\prod_{i \neq j} \mathrm{GL}\left(n_{i}\right) \times Q_{j} \subset \prod_{i} \mathrm{GL}\left(n_{i}\right)=G,
$$

let $\rho$ be a $\sigma$-positive representation of $\check{G}$ where $\sigma$ denotes the product of the characters $\operatorname{det}_{i}: \mathrm{GL}_{n_{i}}(\mathbb{C}) \rightarrow \mathbb{C}^{\times}$. If $x$ is a geometric point of $G-Q$, then

$$
\mathrm{H}_{c}^{*}\left(U_{Q} x, i^{*} \Phi_{\rho}\right)=0
$$

where $i: U_{Q} x \rightarrow G$ denotes the inclusion map. 
Proof. Argue by induction on the semisimple rank of $G$. In the base case $G$ is a torus, hence the proposition holds vacuously.

Otherwise $n_{j} \geq 2$, consider the stratification induced by (4) on $\operatorname{GL}\left(n_{j}\right)$ :

$$
G=\bigsqcup_{m} X_{m}=\bigsqcup_{m=1}^{n_{j}}\left(\prod_{i \neq j} \operatorname{GL}\left(n_{i}\right) \times X_{j, m}\right) .
$$

For $x \notin Q$ with $Q=X_{1}$, we have $x \in X_{m}$ for $2 \leq m \leq n_{j}$.

We first consider the case $x \in X_{n_{j}}$. For $X_{j, n_{j}}$ is contained in the open subset $\operatorname{GL}\left(n_{j}\right)^{\text {reg }}$ of $\operatorname{GL}\left(n_{j}\right)$ (see [S65]), we have a Cartesian diagram

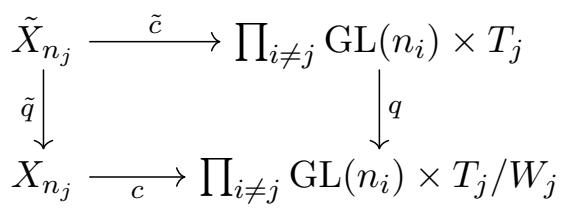

where $c$ is smooth and $q$ is finite. It follows that the restriction of the $\gamma$-sheaf $\Phi_{\rho}$ to $X_{n_{j}}$ can be identified with a pullback by the characteristic polynomial map

$$
\left.\Phi_{\rho}\right|_{X_{n_{j}}}=c^{*} q_{*} \Phi_{\rho^{\prime}} W_{j}
$$

where $\rho^{\prime}$ denotes the restriction of $\rho$ to the subgroup

$$
\prod_{i \neq j} \mathrm{GL}_{n_{i}}(\mathbb{C}) \times \check{T}_{j} \subset \check{G}
$$

We recall that the coordinate ring $T_{j} / W_{j}=\mathbb{A}^{n_{j}-1} \times \mathbb{G}_{m}$ is the ring of $\operatorname{GL}\left(n_{j}\right)$-invariant functions on $\operatorname{GL}\left(n_{j}\right)$, the projection $\sigma_{W_{j}}: T_{j} / W_{j} \rightarrow \mathbb{G}_{m}$ corresponds to the determinant function. By Lemma (4.2), the restriction of $c$ to $U_{Q} x$ induces an isomorphism between $U_{Q} x$ and the fiber of the determinant map on the $j$ th component

$$
\sigma_{W_{j}}: \prod_{i \neq j} \mathrm{GL}\left(n_{i}\right) \times T_{j} / W_{j} \rightarrow \prod_{i \neq j} \mathrm{GL}\left(n_{i}\right) \times \mathbb{G}_{m}
$$

over the image of $x$. Thus, to prove the proposition, it is enough to prove that

$$
\sigma_{!} \Phi_{\rho^{\prime}} W_{j}=\sigma_{W_{j}, !} q_{!} \Phi_{\rho^{\prime}}{ }^{W_{j}}=0
$$

where

$$
\sigma: \prod_{i \neq j} \mathrm{GL}\left(n_{i}\right) \times T_{j} \rightarrow \prod_{i \neq j} \mathrm{GL}\left(n_{i}\right) \times \mathbb{G}_{m}
$$


Proof of Theorem 2.4

is the determinant map on the $j$ th component.

Now recall the definition of the hypergeometric sheaf $\Psi_{\underline{\lambda}}=\mathrm{p}_{\underline{\lambda}}, \operatorname{tr}^{*} \mathcal{L}_{\psi}$ with homorphism $\mathrm{p}_{\underline{\lambda}}: \mathbb{G}_{m}^{r} \rightarrow T$ given by

$$
\mathrm{p}_{\underline{\lambda}}\left(t_{1}, \ldots, t_{r}\right)=\prod_{i=1}^{r} \lambda_{i}\left(t_{i}\right) .
$$

We have

$$
\sigma_{!} \Phi_{\rho^{\prime}}=\sigma_{!} \operatorname{Ind}_{T}^{G^{\prime}} \Psi_{\underline{\lambda}} \prod_{i \neq j} W_{i}
$$

where $G^{\prime}=\prod_{i \neq j} \operatorname{GL}\left(n_{i}\right) \times T_{j}$.

With the definition of the action of $W$ on $\Psi_{\underline{\lambda}}$ given by (2), we see that the induced action of $W$ on $\sigma_{!} \Phi_{\rho^{\prime}}$ is through the character $\operatorname{sign}_{n_{j}}: W_{j} \rightarrow\{ \pm 1\}$. It follows that $\sigma_{!} \Phi_{\rho^{\prime}} W_{j}=0$ because for $n_{j} \geq 2$ the sign character $\operatorname{sign}_{n_{j}}$ is nontrivial. This concludes the case $x \in X_{n_{j}}$.

We now consider the general case $x \in X_{m}$ with $2 \leq m \leq n_{j}$. By $Q$ conjugation we can assume that the $j$ th component $x_{j}$ of $x$ is of the form (16) with $x_{j, F}$ being a companion matrix as in (5). Let $P$ denote the standard parabolic of block matrices as in (6),$L$ the standard Levi factor of $P$ and $U_{P}$ its unipotent radical. By applying the result obtained above in the generic case to $\mathrm{GL}(F)$, we get

$$
\mathrm{H}_{c}^{*}\left(U_{L} x_{L},\left.\Phi_{L, \rho \mid \check{L}}\right|_{U_{L} x_{L}}\right)=0
$$

where $x_{L}$ is the image of $x$ in $L, U_{L}$ consists of unipotent matrices of the form (7), and $\Phi_{L,\left.\rho\right|_{\check{L}}}$ is the $\gamma$-sheaf on $L$ associated to the restriction to $\check{L}$ of the representation $\rho$ of $\check{G}$.

For $\Phi_{L,\left.\rho\right|_{\breve{L}}}=\operatorname{Res}_{L}^{G}\left(\Phi_{\rho}\right)$ by Proposition 2.2, (8) implies that

$$
\mathrm{H}_{c}^{*}\left(U_{P} U_{L} x,\left.\Phi_{\rho}\right|_{U_{P} U_{L} x}\right)=0
$$

where $U_{P}$ and $U_{L}$ commute. With the help of Lemma 3.2, we see that the morphism

$$
U_{Q} \times U_{m-1} \rightarrow U_{P} U_{L} x
$$

given by

$$
\left(u_{m-1}, u_{Q}\right) \mapsto u_{m-1} u_{Q} x u_{m-1}^{-1}
$$

is an isomorphism. Now using the fact that $\Phi_{\rho}$ is equivariant under the adjoint action, (9) implies that

$$
\mathrm{H}_{c}^{*}\left(U_{Q} x,\left.\Phi_{\rho}\right|_{U_{Q} x}\right)=0 .
$$

This concludes the proof of Proposition 5.1 and therefore Theorem 2.4. 
The case of parabolic subgroups

\section{A The case of parabolic subgroups}

In this appendix we give a proof for the extension of Theorem 2.4 to arbitrary parabolic subgroups $P$ of $G=\mathrm{GL}(n)$. By a similar argument involving the Leray spectral sequence as in the beginning of the proof of Theorem 2.4, we are reduced to the case when $P$ is a maximal parabolic subgroup.

Proposition A.1. Let $P$ denote the maximal standard parabolic subgroup of $G=\mathrm{GL}(n)$ consisting of block matrices of size $\left(n_{1}, n_{2}\right)$ where $n_{1}+n_{2}=n$ and $U_{P}$ its unipotent radical, let $\rho$ be a $\sigma$-positive representation of $\breve{G}$ where $\sigma=$ det. If $g$ is a geometric point of $G-P$, then

$$
\mathrm{H}_{c}^{*}\left(U_{P} g, i^{*} \Phi_{\rho}\right)=0
$$

where $i: U_{P} g \rightarrow G$ denotes the inclusion map.

Proof. Argue by induction on $n_{1}$. In the base case when $n_{1}=1$ we are reduced to Proposition 5.1 .

Otherwise $n_{1} \geq 2$, let $P^{\prime}$ denote the maximal standard parabolic subgroup of $\mathrm{GL}(n)$ consisting of block matrices of size $\left(n_{1}-1, n_{2}+1\right)$ and $U_{P^{\prime}}$ its unipotent radical. Let

$$
g^{\prime}=\left[\begin{array}{l|l}
a & b \\
\hline c & d
\end{array}\right]
$$

be a block matrix of size $\left(n_{1}, n_{2}\right)$ such that the $n_{2} \times n_{1}$-matrix $c$ is of the form

$$
b=\left[\begin{array}{ccc|c}
* & \cdots & * & 1 \\
\hline * & \cdots & * & 0 \\
\vdots & \ddots & \vdots & \vdots \\
* & \cdots & * & 0
\end{array}\right],
$$

then by an analogous computation as in the proof of Lemma 3.2 and the end of the proof of Proposition 5.1, we see that acyclicity of $\Phi_{\rho}$ over $U_{P^{\prime}} g$ :

$$
\mathrm{H}_{c}^{*}\left(U_{P^{\prime}} g^{\prime},\left.\Phi_{\rho}\right|_{U_{P^{\prime}} g^{\prime}}\right)=0
$$

implies acyclicity over the subcoset $\left(U_{P} \cap U_{P^{\prime}}\right) g$ :

$$
\mathrm{H}_{c}^{*}\left(\left(U_{P} \cap U_{P^{\prime}}\right) g^{\prime},\left.\Phi_{\rho}\right|_{\left(U_{P} \cap U_{P^{\prime}}\right) g^{\prime}}\right)=0,
$$

which then implies the proposition by the Levy spectral sequence.

Now it remains to prove (10). To this end it suffices to observe that $g$ is conjugate, under the action of the standard Levi factor $L$ of $P$, to a matrix of the form $g^{\prime}$, which is in addition not contained in $P^{\prime}$. This follows from the fact that the $L$-orbits on $c$ are classified by the rank of $c$. Hence we are done by induction. 
Positive hypergeometric sheaves

\section{B Positive hypergeometric sheaves}

In this appendix we give a proof for Proposition 1.1 concerning hypergeometric sheaves $\Psi_{\underline{\lambda}}$ when $\underline{\lambda}$ is $\sigma$-positive. The first half of Proposition 1.1 is due to Braverman-Kazhdan in [BK02, Theorem 4.2]:

Proposition B.1. If $\underline{\lambda}$ is $\sigma$-positive, then the forget support morphism $\Psi_{\underline{\lambda}} \rightarrow \Psi_{\underline{\lambda}}^{*}$ is an isomorphism.

This is proved by restricting to the smooth neighborhood $\mathrm{p}_{\underline{\lambda}}: \mathbb{G}_{m}^{r} \rightarrow T$ and then applying the classical Fourier-Deligne transform on $\mathbb{G}_{a}^{r}$. The same idea is also crucial in the proof of the second half of Proposition 1.1;

Proposition B.2. If $\underline{\lambda}$ is $\sigma$-positive, then $\Psi_{\underline{\lambda}}$ is isomorphic to a shift of a local system on the image of $\mathrm{p}_{\underline{\lambda}}: \mathbb{G}_{m}^{r} \rightarrow T$.

Without loss of generality we can assume that $\mathrm{p}_{\underline{\lambda}}$ is surjective. Then we can also factorize $\mathrm{p}_{\underline{\lambda}}$ into the product of a homomorphism with connected fibers and an isogeny. Since isogenies preserve local systems under pushforward by proper-smooth base change, without loss of generality we can assume that $\mathrm{p}_{\underline{\lambda}}$ has connected fibers.

We will deduce smoothness of $\Psi_{\underline{\lambda}}$ from universal local acyclicity by Théorèm 5.3.1 in [D77a] together with the fact that $\Psi_{\underline{\lambda}}$ is a perverse sheaf by Proposition B.1, for this we need a compactification of $\mathrm{p}_{\underline{\lambda}}$. Let $\bar{\Gamma}$ be the normalization of the closure of the graph of $\mathrm{p}_{\underline{\lambda}}$ in $\left(\mathbb{G}_{m}\right)^{r} \times \bar{T} \subset\left(\mathbb{P}^{1}\right)^{r} \times T$, let $\mathrm{j}_{\underline{\lambda}}:\left(\mathbb{G}_{m}\right)^{r} \rightarrow \bar{\Gamma}$ be the open inclusion and $\overline{\mathrm{p}}_{\underline{\lambda}}: \bar{\Gamma} \rightarrow T$ the projection, then $\overline{\mathrm{p}}_{\underline{\underline{\lambda}}}$ compactifies $\mathrm{p}_{\underline{\lambda}}$.

We are therefore reduced to the following lemma:

Lemma B.3. If $\underline{\lambda}$ is $\sigma$-positive and $\mathrm{p}_{\underline{\lambda}}$ is surjective with connected fibers, then $\overline{\mathrm{p}}_{\underline{\lambda}}$ is universally locally acyclic with respect to $\mathrm{j}_{\underline{\lambda}}, ! \mathrm{tr}^{*} \mathcal{L}_{\psi}$.

Proof. The following argument is essentially due to Katz-Laumon in [KL85].

Let $\pi:\left(\mathbb{P}^{1}\right)^{r} \rightarrow\left(\mathbb{P}^{1}\right)^{r}$ be the completed Artin-Schreier covering defined by $[X: Y] \mapsto\left[X^{q}-X Y^{q-1}: Y^{q}\right]$ on the homogeneous coordinates on each factor $\mathbb{P}^{1}$ and $\tilde{\pi}$ the base change of $\pi$ along the projection $\bar{\Gamma} \rightarrow\left(\mathbb{P}^{1}\right)^{r}$. It follows from the decomposition theorem and total ramification of $\pi$ at infinity that the !-extension of $\operatorname{tr}^{*} \mathcal{L}_{\psi}$ to $\left(\mathbb{P}^{1}\right)^{r}$ is a direct summand of $\pi_{*} \overline{\mathbb{Q}}_{\ell}$ over $\left(\mathbb{P}^{1}\right)^{r}-(0, \ldots, 0)$.

Now by assumption $\underline{\lambda}$ is $\sigma$-positive, hence the image of $\bar{\Gamma}$ in $\left(\mathbb{P}^{1}\right)^{r}$ is contained in $\left(\mathbb{P}^{1}\right)^{r}-(0, \ldots, 0)$ which implies that $\mathrm{j}_{\underline{\lambda}}, \mathrm{tr}^{*} \mathcal{L}_{\psi}$ is a direct summand of $\tilde{\pi}_{*} \overline{\mathbb{Q}}_{\ell}$. Therefore it suffices to show instead that $\overline{\mathrm{p}}_{\underline{\lambda}}$ is universally locally acyclic with respect to $\tilde{\pi}_{*} \overline{\mathbb{Q}}_{\ell}$. 
Positive hypergeometric sheaves

Let $K$ be the kernel of $\mathrm{p}_{\underline{\lambda}}$ which we may assume to be connected, let $\bar{K}$ be the normalization of the closure of $K$ in $\left(\mathbb{P}^{1}\right)^{r}$ and $\bar{\mu}:\left(\mathbb{G}_{m}\right)^{r} \times \bar{K} \rightarrow \bar{\Gamma}$ the morphism which extends the multiplication map from $\left(\mathbb{G}_{m}\right)^{r} \times K$ to $\left(\mathbb{G}_{m}\right)^{r}$. Then the diagram

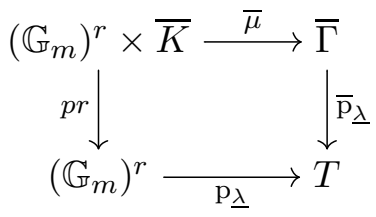

is Cartesian by the theory of toric varieties (see [F93] for example). Let $\hat{\pi}$ be the base change of $\tilde{\pi}$ along $\bar{\mu}$.

We may assume that $\mathrm{p}_{\lambda}$ is surjective hence smooth. Since universal local acyclicity is a local property with respect to the smooth topology, it suffices to show that $p r$ is universally locally acyclic with respect to $\hat{\pi}_{*} \overline{\mathbb{Q}}_{\ell}$. Then by properness of $\hat{\pi}$ we are further reduced to showing that the composite $p r \circ \hat{\pi}$ is universally locally acyclic with respect to the constant sheaf $\overline{\mathbb{Q}}_{\ell}$ on the source.

Let $U$ be an open subscheme of $\left(\mathbb{P}^{1}\right)^{r}$ of the form $U=U_{1} \times \cdots \times U_{r}$ where each Cartesian factor $U_{i}$ is an open subscheme of $\mathbb{P}^{1}$ equal to either $\mathbb{G}_{a}$ or $\mathbb{P}^{1}-0$. Let $\widetilde{U}$ be the inverse image of $U$ under $\pi:\left(\mathbb{P}^{1}\right)^{r} \rightarrow\left(\mathbb{P}^{1}\right)^{r}$ and $\bar{K}_{U}$ the intersection of $\bar{K}$ with $U$. We will check universal local acyclicity of $p r \circ \hat{\pi}$ by restricting to the open subscheme $\widetilde{U} \times_{U}\left(\left(\mathbb{G}_{m}\right)^{r} \times \bar{K}_{U}\right)$ of the source of $\hat{\pi}$ and then calculating "by hand" with explicit coordinates.

To this end let $t$ be a coordinate on $\mathbb{G}_{m}, X$ a coordinate on $\mathbb{G}_{a}$ and $Y^{-1}$ a coordinate on $\mathbb{P}^{1}-0$, hence $\left(\ldots, X_{i}, \ldots, Y_{j}^{-1}, \ldots\right)$ are coordinates on $U$. By slight abuse of notation let $\mathrm{p}_{\underline{\lambda}}\left(\ldots, X_{i}, \ldots, Y_{j}^{-1}, \ldots\right)=1$ denote a system of polynomial equations such that

$$
\bar{K}_{U} \simeq \operatorname{Spec}\left(\overline{\operatorname{Int}}\left(\frac{k\left[\ldots, X_{i}, \ldots, Y_{j}, \ldots\right]}{\left(\mathrm{p}_{\underline{\underline{\lambda}}}\left(\ldots, X_{i}, \ldots, Y_{j}^{-1}, \ldots\right)=1\right)}\right)\right)
$$

where $\overline{\text { Int }}$ denotes the integral closure, then modulo base change with respect to a normalization, we have that $\underset{U}{\widetilde{U}}\left(\left(\mathbb{G}_{m}\right)^{r} \times \bar{K}_{U}\right)$ is isomorphic to

$$
\operatorname{Spec}\left(\frac{k\left[\begin{array}{c}
\ldots, t_{n}, t_{n}{ }^{-1}, \ldots, X_{i}, \ldots, Y_{j}, \ldots \\
\ldots, \widetilde{X}_{i}, \ldots, \widetilde{Y}_{j},\left(1-\widetilde{Y}_{j}^{q-1}\right)^{-1}, \ldots
\end{array}\right]}{\left(\begin{array}{c}
\widetilde{X}_{i}^{q}-\widetilde{X}_{i}=t_{i} X_{i},\left(1-\widetilde{Y}_{j}^{q-1}\right)^{-1} \widetilde{Y}_{j}^{q}=t_{j}{ }^{-1} Y_{j}, \\
\mathrm{p}_{\underline{\underline{x}}}\left(\ldots, X_{i}, \ldots, Y_{j}^{-1}, \ldots\right)=1
\end{array}\right)}\right),
$$


which by substituting $s_{j}$ for $\left(1-\widetilde{Y}_{j}^{q-1}\right)^{-1} t_{j}$ becomes isomorphic to

$$
\operatorname{Spec}\left(\frac{k\left[\begin{array}{c}
\ldots, t_{i}, t_{i}^{-1}, \ldots, s_{j}, s_{j}^{-1}, \ldots \\
\ldots, \widetilde{X}_{i}, \ldots, \widetilde{Y}_{j},\left(1-\widetilde{Y}_{j}^{q-1}\right)^{-1}, \ldots
\end{array}\right]}{\left(\mathrm{p}_{\underline{\underline{\lambda}}}\left(\ldots, t_{i}^{-1}\left(\widetilde{X}_{i}^{q}-\widetilde{X}_{i}\right), \ldots,\left(s_{j} \widetilde{Y}_{j}^{q}\right)^{-1}, \ldots\right)=1\right)}\right) .
$$

The morphism $\widetilde{X} \mapsto \widetilde{X}^{q}-\widetilde{X}$ is étale and the morphism $\widetilde{Y} \mapsto \widetilde{Y}^{q}$ is finite surjective radicial, both are universally locally acyclic with respect to $\overline{\mathbb{Q}}_{\ell}$ on the source. By Corollaire 2.16 in [D77f] the projection $p r$ from

$$
\operatorname{Spec}\left(\overline{\operatorname{Int}}\left(\frac{k\left[\ldots, t_{n}, t_{n}{ }^{-1}, \ldots, X_{i}, \ldots, Y_{j}, \ldots\right]}{\left.\left(\mathrm{p}_{\underline{\underline{\lambda}}}\left(\ldots, t_{i}{ }^{-1} X_{i}, \ldots, t_{j} Y_{j}\right)^{-1}, \ldots\right)=1\right)}\right)\right) \simeq\left(\mathbb{G}_{m}\right)^{r} \times \bar{K}_{U}
$$

to $\left(\mathbb{G}_{m}\right)^{r}$ is universally locally acyclic with respect to $\overline{\mathbb{Q}}_{\ell}$ on the source. Hence so is their composite, the lemma follows.

\section{References}

[BBD82] A. Beilinson, J. Bernstein and P. Deligne, Faisceaux pervers, in Analysis and topology on singular spaces, I (Luminy 1981), Astérisque, 100 (1982), 5-171.

[B84] J. Bernstein, P-invariant distributions on $G L(N)$ and the classification of unitary representations of $G L(N)$ (nonArchimedean case). in Lie group representations, II (College Park, Md., 1982/1983), 50102, Lecture Notes in Math., 1041, Springer, Berlin, 1984.

[BK00] A. Braverman and D. Kazhdan, $\gamma$-functions of representations and lifting (with an appendix by V. Vologodsky), Geom. Funct. Anal., Special Volume (2002), Part I, 237-278.

[BK02] A. Braverman and D. Kazhdan, $\gamma$-sheaves on reductive groups, in Studies in memory of Issai Schur (Chevaleret/Rehovot 2000), Progr. Math., 210 (2003), 27-47.

[D77a] P. Deligne, Cohomologie étale: les points de départ, in Cohomologie étale (SGA 4 1/2), Lecture Notes in Math., 569 (1977), 4-75. 
[D77s] P. Deligne, Applications de la formule des traces aux sommes trigonométriques, in Cohomologie étale (SGA 4 1/2), Lecture Notes in Math., 569 (1977), 168-232.

[D77f] P. Deligne, Théorèmes de finitude en cohomologie $\ell$-adique, in Cohomologie étale (SGA 4 1/2), Lecture Notes in Math., 569 (1977), 233-261.

[F93] W. Fulton, Introduction to toric varieties, Ann. of Math. Stud., 131 (1993).

[GJ72] R. Godement and H. Jacquet, Zeta functions of simple algebras, Lecture Notes in Math., 260 (1972).

[KL85] N. Katz and G. Laumon, Transformation de Fourier et majoration de sommes exponentielles, Publ. Math. IHES, 62 (1985), 145-202.

[K90] N. Katz, Exponential sums and differential equations, Ann. of Math. Stud. 124(1900), 1-430.

[L13] L. Lafforgue, Noyaux du transfert automorphe de Langlands et formules de Poisson non linéaires, Preprint, 2013, http://www.ihes.fr/ lafforgue/publications.html.

[T50] J. Tate, Fourier analysis in number fields, and Hecke's zetafunctions, in Algebraic Number Theory, Academic Press, New York, 1967, 305-347.

[S65] R. Steinberg, Regular elements of semisimple algebraic groups, Publ. Math. IHES, 25 (1965), 49-80. 